\title{
EFFECT OF DIFFERENT SURFACE TREATMENT PROTOCOLS ON THE RETENTION OF POSTERIOR PEEK CROWNS (A RANDOMIZED INVITRO STUDY)
}

\author{
Kareem Mohamed Abdelmotaleb ${ }^{*}$, Hesham Katamesh ${ }^{* *}$ and Reham Saeed El-Basty ${ }^{* * *}$
}

\begin{abstract}
Aim: The purpose of this study is to evaluate the retention of PEEK-based posterior crowns after different surface treatment protocols (sandblasting using 110 micron alumina particles, acid etching using sulphuric acid and combined sandblasting and acid etching).

Methodology: 24 extracted human natural molars that were mounted in epoxy blocks and they were prepared to receive PEEK copings that were divided into 3 groups. The first group was treated with sandblasting with 110 micron alumina particles under 2 bar, The second group was treated with $98 \%$ sulphuric acid for 60 seconds then rinsed with running water for 60 seconds, The third group was treated with sandblasting and acid etching together then all copings were cemented to their corresponding teeth with Relyx Ultimate clicker then after cementation all copings were subjected to a pull out test using a Universal testing machine. After the mesurments the teeth were examined under digital microscope to detect the mode of failure.
\end{abstract}

Results: For Sandblasted group the mean $\pm \mathrm{SD}$ values were $(80.66 \pm 5.54 \mathrm{~N})$ with minimum value $(59.31 \mathrm{~N})$ and maximum value $(108.27 \mathrm{~N})$, while for Acid etched group the mean $\pm \mathrm{SD}$ values were $(87.45 \pm 5.56 \mathrm{~N})$ with minimum value $(81.96 \mathrm{~N})$ and maximum value $(101.34 \mathrm{~N})$. Meanwhile the mean \pm SD values for Sandblasted and Acid etched group together were $(92.43 \pm 5.51 \mathrm{~N})$ with minimum value $(79.67 \mathrm{~N})$ and maximum value $(106.19 \mathrm{~N})$. In accordance to the mode of failure In $\mathrm{SB}$ and $\mathrm{AE}$ treated group, the failure mode pattern was predominantly adhesive while minority was mixed. In $\mathrm{SB}+\mathrm{AE}$ treated group samples showed equal \% between adhesive and mixed failure mode patterns. There was no record for cohesive failure mode pattern in all groups.

Conclusions: Within the limitation of this study; The surface treatment that was preformed by using sandblasting and sulphuric acid etching togethere showed the highest results in accordance to retention of the copings. The use of different surface treatment protocols gives a reliable results in accordance to retention.

KEYWORDS: Retention, Posterior Peek.

\footnotetext{
* Master of Fixed Prosthodontics, Cairo University

** Professor at Fixed Prosthodontics Department, Faculty of Dentistry Cairo University.

*** Associate Professor of Fixed Prosthodontics, Faculty of Dentistry Cairo University.
} 


\section{INTRODUCTION}

Due to the increased aesthetic demands, new materials are always being introduced to combine aesthetics with proper mechanical properties and cost effectiveness. Recently Polyetheretherketone (PEEK) was introduced to be a potentially promising material in the field of fixed prosthodontics.

The major advantage of PEEK is its $4 \mathrm{GPa}$ modulus of elasticity (stress breaking and shock absorbing) in combination with its unique mechanical and biological properties; making it highly desirable as a FDP framework material.

Moreover, PEEK exhibits low density (1.3-1.5 $\mathrm{gm} / \mathrm{cm}^{3}$ ) which leads to lighter restorations, more comfort and feeling of a foreign body after initial tryin is eliminated. Moreover, PEEK material surface properties is very promising. It exhibits a polished surface which is crucial for biofilm formation and bacterial plaque accumulation.

PEEK is biocompatible and features a natural tooth coloured appearance as compared to metal reconstructions. However, from the aesthetic point of view, it still requires veneering owing to its low translucency and greyish, greyish-brown or pearlwhite opaque colour and is unsuitable for monolithic aesthetic dental restorations, so they cannot be milled to full contour.

PEEK can be veneered with CAD/CAM milled composite blanks using methyl-methacrylate-based bonding to achieve an adequate bond strength.

Therefore, PEEK has the potential to be a core material for fabrication of aesthetic restorations if proven to be comparable to zirconia. And this study was conducted to compare the fracture resistance and mode of failure of Zirconia core veneered by e.max veneer to PEEK core veneered by High Impact Polymer Composite

\section{MATERIALS AND METHODS}

\section{Teeth selection}

24 extracted intact molar teeth were selected. Teeth were disinfected using 1:10 sodium hypochlorite solution and stored in $0.9 \%$ saline solution. They were then mounted in epoxy resin molds* using a paralleling device Fig.(1).

Measurements of the mesiodistal and buccolingual dimensions of the teeth were taken using a digital caliper. It was ensured that the teeth had close measurements to standardize the bonding surface area of the teeth substrate.

\section{Teeth preparation}

Preparation of the teeth was done to receive milled PEEK copings. Silicone indicies for teeth before preparation were fabricated and sectioned in a buccolingual direction.

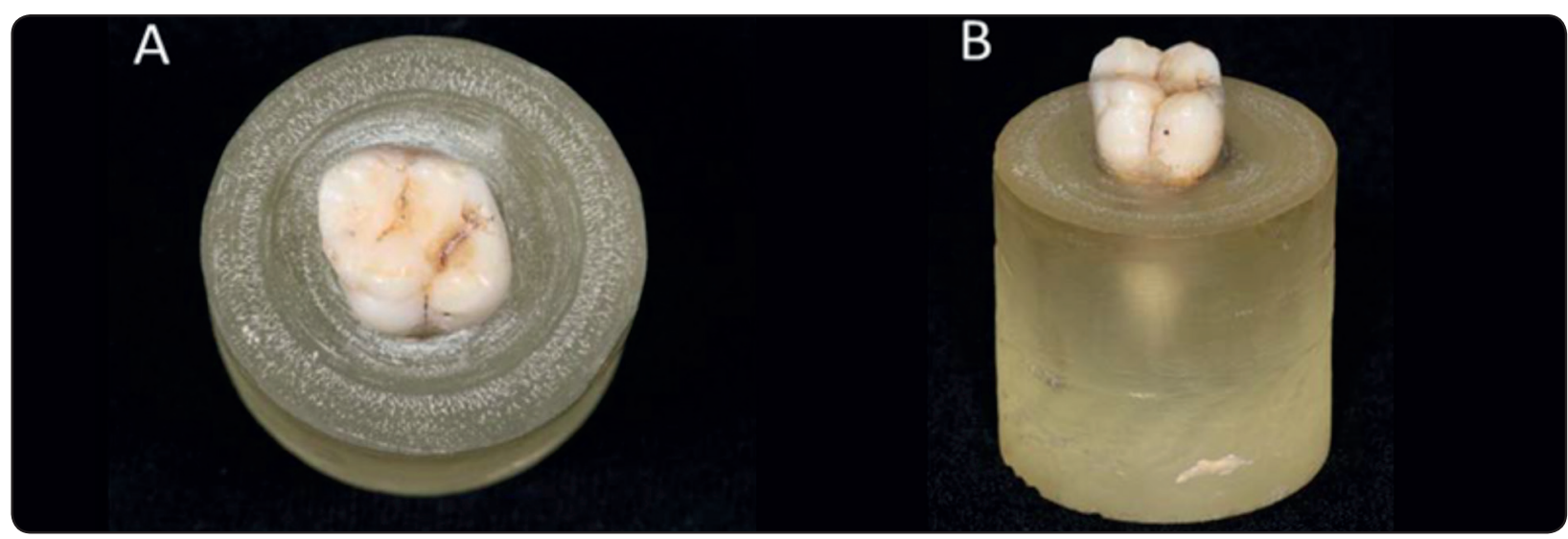

Fig. (1) : Extracted molar teeth mounted in epoxy resin molds 
Axial preparation was done with blue grit tapered stone with rounded end to obtain deep chamfer finish line $1 \mathrm{~mm}$ in width. Preparation followed the anatomy of the teeth. Axial surfaces were prepared using a milling machine* with an attached straight hand piece

\section{Data acquisition and designing}

Prepared teeth were scanned using an extra oral scanner Figure (2) to produce virtual dies. The full coverage copings were designed using a design software (Exo CAD). A single horizontal bar was designed occlusally to allow engagement of orthodontic wires in the pull out test.

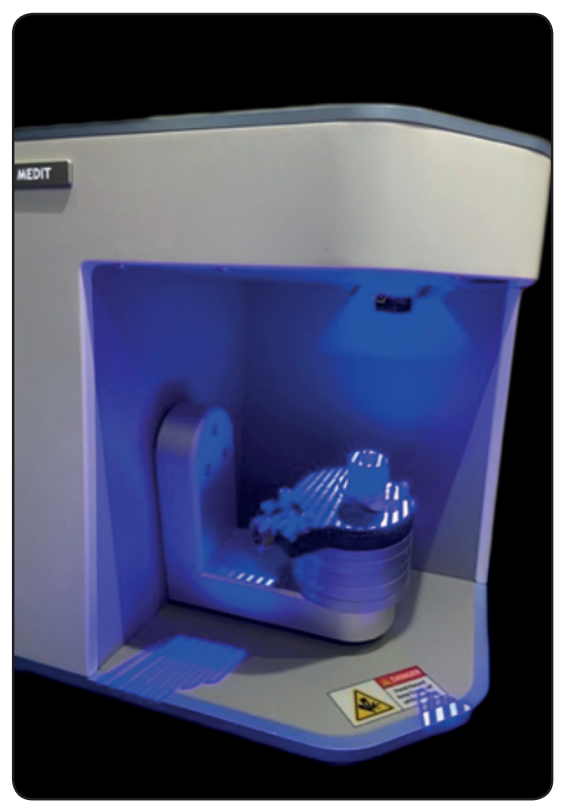

Fig. (2) Scanning of the prepared teeth

\section{Construction of PEEK copings}

PEEK copings were milled by a 5 Axis milling machine

\section{Teeth Grouping}

PEEK copings $(n=24)$ were divided into 3 main groups according to the surface treatment protocol Table 3. In The first group $(n=8)$, the surface treatment used was sand blasting. In The second group ( $\mathrm{n}=8)$ acid etching with sulphuric acid. The third group $(n=8)$ the surface treatment used was sandblasting and acid etching.

\section{Surface treatment}

\section{For the first group}

A special holder was manufactured to standardize sandblasting of the copings. The copings were sandblasted with 110 micron alumina under 2 bar and at $10 \mathrm{~mm}$ distance.

\section{For the second group}

The coping were etched with sulphuric acid $98 \%$ for 60 seconds and then rinsed with running water for another 60 seconds and then dried with oil free air.

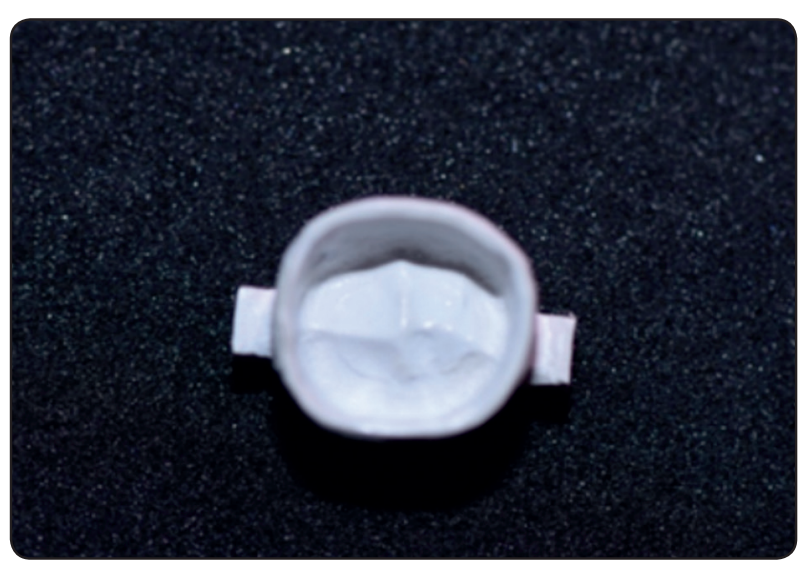

Fig. (3): Fitting surface after acid etching

\section{For the third group}

The copings were sandblasted with alumina 110 micron under 2 bar at $10 \mathrm{~mm}$ distance and then etched with sulphuric acid $98 \%$ for 60 seconds and then rinsed under running water for another 60 seconds and then dried with oil free air .

\section{Bonding procedures}

The prepared teeth were rinsed with water followed by drying with oil free air. Since the preparation was totally within dentin, universal 
adhesive (Scotchbond Universal) was applied to the prepared teeth using a microbrush and scrubbed for 20 secs, air thinned with a gentle stream of oil free air for 5 seconds and left without curing.

The copings were seated first with finger pressure on the teeth. Tack curing for $1 \mathrm{sec}$ on each surface was done where the excess cement reached a rubber like consistency that facilitated its removal using a manual scaler.

A load of $3 \mathrm{kgs}$ was applied axially on the coping through placing it in a cementing device. It consists of a base on which the epoxy resin mould was placed. The selected weight was then placed on a piston that was applied perpendicular to the occlusal surface of the crown and along its long axis keeping it under compressive load until complete curing of the cement after 20 mins. Light curing unit of 1200 $\mathrm{mW} / \mathrm{cm} 2$ was used for curing each surface for 40 seconds

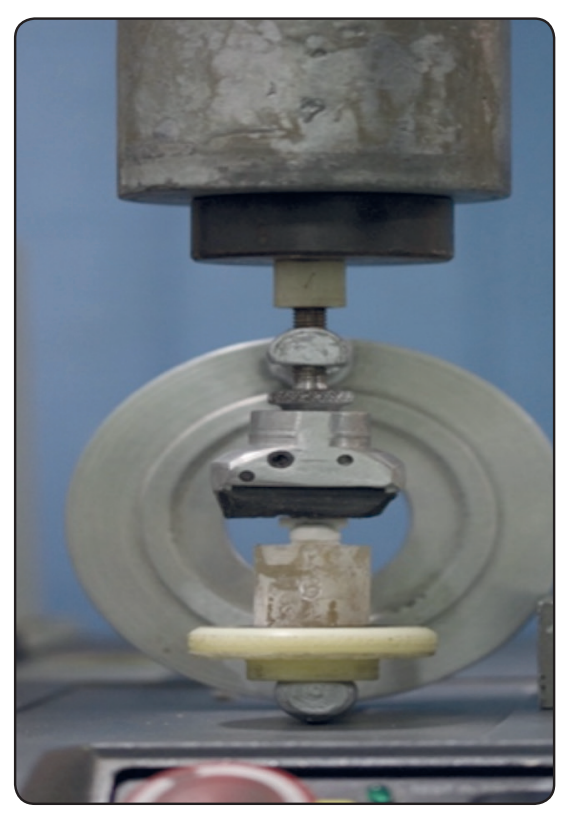

Fig. (4): Cementing device

\section{Pull out test}

Retention was measured using Materials Testing Machine with a load cell of $5 \mathrm{KN}$. Data were recorded using computer software. The copings was suspended from the upper movable compartment of the testing machine by double orthodontic wire loop $(0.7 \mathrm{~mm})$ through the occlusal bar made during milling. The device was subjected to a slowly increasing vertical load $(1 \mathrm{~mm} / \mathrm{min})$ until failure. The load required to dislodgment was recorded in Newton.

\section{Mode of failure.}

The mode of failure (adhesive, cohesive or mixed) was detected using a digital microscope. The images were captured and then transferred to IBM personal computer equipped with the Imagetool software.

\section{RESULTS:}

The results were analyzed using Graph Pad Instat (Graph Pad, Inc.,USA) software for windows. A value of $\mathrm{P}<0.05$ was considered statistically significant. After homogeneity of variance and normal distribution of errors had been confirmed, one-way ANOVA was done for comparison between groups followed by Tukey's pairwise if showed significant results. Sample size ( $\mathrm{n}=8$ /group) was large enough to detect large effect sizes for main effects and pair-wise comparisons, with the satisfactory level of power set at $80 \%$ and a $95 \%$ confidence level.

\section{Retention}

For SB treated group the mean \pm SD values were $(80.66 \pm 5.54 \mathrm{~N})$ with minimum value $(59.31 \mathrm{~N})$ and maximum value $(108.27 \mathrm{~N})$, while for $\mathrm{AE}$ treated group the mean $\pm \mathrm{SD}$ values were $(87.45 \pm 5.56 \mathrm{~N})$ with minimum value $(81.96 \mathrm{~N})$ and maximum value $(101.34 \mathrm{~N})$. Meanwhile the mean $\pm \mathrm{SD}$ values for $\mathrm{AE}+\mathrm{SB}$ treated group were $(92.43 \pm 5.51 \mathrm{~N})$ with minimum value $(79.67 \mathrm{~N})$ and maximum value $(106.19 \mathrm{~N})$.

It was found that $\mathrm{AE}+\mathrm{SB}$ treated group recorded the highest retention mean value $(92.43 \pm 5.51 \mathrm{~N})$ 
followed by AE treated group $(87.45 \pm 5.56 \mathrm{~N})$ while SB treated group recorded the lowest retention mean value $(80.66 \pm 20.64 \mathrm{~N})$.

The difference between all groups was statistically non-significant $(\mathrm{p}=0.2033>0.05)$ as indicated by ANOVA test.

\section{Mode of failure}

Frequent distribution of failure modes scores (\%) for all groups are summarized in table (6) and graphically drawn in figure (33).

In $\mathrm{SB}$ and $\mathrm{AE}$ treated group, the failure mode pattern was predominantly adhesive while minority was mixed. Figure (34-35)

In $\mathrm{SB}+\mathrm{AE}$ treated group samples showed equal $\%$ between adhesive and mixed failure mode patterns. There was no record for cohesive failure mode pattern in all groups.

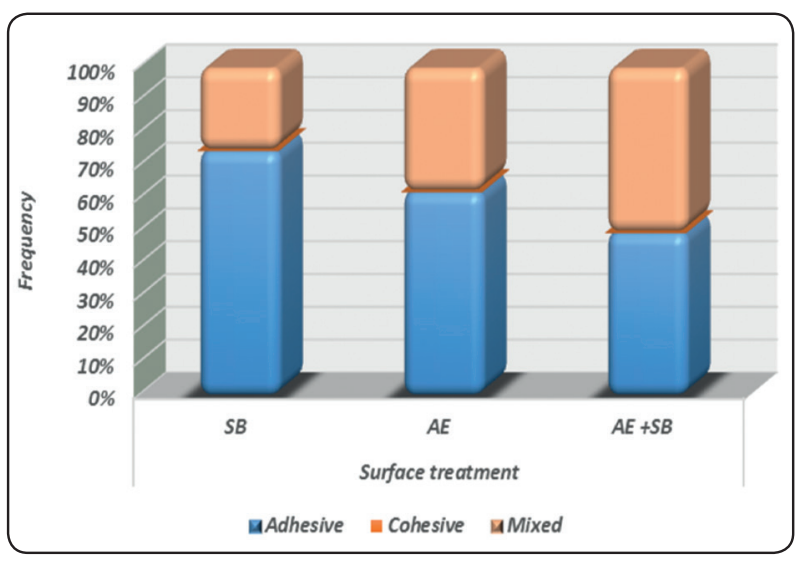

Fig. (5): Stacked column chart comparing the frequent distribution of failure modes scores for all groups

\section{DISCUSSION}

PEEK is a new promising evolving material in the field of dentistry with huge advantages. It has a modulus of elasticity $4 \mathrm{GPa}$ which is very close to that of bone and dentin thus it transfers the least amount of stresses and can have a shock absorbing action. Moreover, it is claimed that it has high strength values; fracture resistance around $2000 \mathrm{~N}$ and flexural strength >180 MPa. In addition biologically, PEEK material surface properties is very promising with very low biofilm formation. ${ }^{(10,11)}$

Due to this advantages PEEK has became an interesting and competitive candidate with ceramics in fixed dental prosthesis. ${ }^{(17)}$

The resistance of a crown to dislodgement (retention to the underlying abutment) is an important prerequisite for its long term survival. It was also noted as the main reason for the failure of single crown restoration ${ }^{(19,20)}$

Testing the mechanical properties of PEEK is quite overlooked in the literature. So this study was conducted with the aim of comparing the effect of different surface treatment protocols on the retention of PEEK copings to their corresponding natural teeth abutments.

Freshly extracted human posterior molars were chosen for the study with average dimensions in order to standardize the study factors as much as possible. Many studies used stainless steel or resin dies for the retention testing of crowns as they include standardized preparation and identical physical quality of materials used. However, prepared teeth made of steel do not reproduce the real force distribution that occur on the crowns cemented on natural teeth. Dentin has a lower elastic modulus than steel; therefore, the inner crown surface shows a greater shear stress every time the tooth is subjected to deformation. Also the wetness of the dentin and its thickness are influen $\neg$ tial variables in in-vitro studies that attempt to imitate in-vivo condition. ${ }^{(1)}$

Teeth were mounted in epoxy resin blocks using a parallel-meter in order to ensure a standardized tooth mounting angulation. This was done to avoid tilting during mounting to avoid any unneeded forces that could affect the pull out testing procedures.

Teeth were prepared by the same operator using a standardized technique. A special surveying milling 
machine with a properly chosen stone was used for gross preparation to produce the same amount of reduction with the same degree of taper which was carefully checked afterwards. The preparation parameters were checked being $1 \mathrm{~mm}$ deep chamfer finish line, $1.5 \mathrm{~mm}$ axial reduction with 100 taper and $2 \mathrm{~mm}$ occlusal reduction. Those parameters were selected following the manufacturer's recommendations.

$\mathrm{CAD} / \mathrm{CAM}$ milling was used in order to achieve better precision of the constructed crowns.

Also; fabricated PEEK CAD/CAM blocks produce restorations with better mechanical and optical properties than those thermo-pressed from granular or pellet-shaped PEEK. ${ }^{(16,21)}$

A 5-axis milling machine was chosen as it was suggested by many studies and claimed by many authors to give better and a highly accurate restoration dimensions, which would result in better internal, marginal fit, and in turn affect retention of the final restoration. ${ }^{(2,9)}$

Concerning the surface treatment for the three groups, sandblasting with $110 \mu \mathrm{m}$ alumina particles under 2 bar and 98\% sulphuric acid etching for 60 seconds was done according to manufactures instructions and also according to many studies that claimed that those surface treatment protocols gives us the highest bond strength compared to other surface treatments like laser etching and plasma treatment. ${ }^{(15,18,4)}$

As regards the bonding procedures and adhesive resin cement was used according to the manufactures instructions.

The chosen cement RelyX ultimate contains MMA monomer. Many authors claimed that MMA containing adhesive cements increases the bond strength of the PEEK restoration to the underlying substrate. ${ }^{(15,18)}$

Problems in the cement layer like voids, discontinuity, inadequate thickness and improper base/catalyst ratio affect the mechanical properties of the cement and the long-term bond strength ${ }^{(12}$, ${ }^{14)}$.Hence, the RelyX ${ }^{\mathrm{TM}}$ Ultimate clicker dispenser system was preferred to provide equal base/catalyst proportions.

Also, a homogenous cement mix was done with care using a stainless-steel spatula, and evenly distributed on the crowns intaglios using a penbrush. Dual curing was also shown to significantly improve the bond strength between resin cement and zirconia Y-TZP ${ }^{(13)}$ accordingly, dual cured cement was used with PEEK crowns.

To apply a consistent seating pressure during cementation of all crowns, a specially designed device with a 3-kg weight was used, as recommended by Karimipour-Saryazdi et al. ${ }^{(7)}$. That was performed as authors believed that seating pressure can influence the internal adaptation and the final strength of adhesive resin cements; and hence retention ${ }^{(3)}$

According to the retention test, application of tensile pull-out force on a crown is considered a logical, reproducible and reliable method to test the retentive strength of a crown to its underlying abutment which simulates the clinical situation that occurs in the real life as stated by Heintze ${ }^{(5)}$; Keul et al. ${ }^{(8)}$

An occlusal bar was designed in the crown to hold the wires for the retention test as recommended by Johnson et al. ( ${ }^{(\boldsymbol{)}}$. A perfectly horizontal base of the epoxy die, the exactly occlusal bar, and a tightly fixed specimen to the universal testing machine having the pulling part of the universal testing machine centralized over it; were the considered criteria for avoiding the shearing forces during crown dislodgment throughout the retention test ${ }^{(5)}$

Regarding the effect of different surface treatment protocols on the retention of PEEK copings, results showed that PEEK copings group that were treated by sandblasting and sulphuric acid etching recorded a mean retention value of $(92.43 \mathrm{~N})$ while PEEK copings group that were treated by sandblasting recorded a mean retention value of $(80.66 \mathrm{~N})$ and 
PEEK copings group that were treated by sulphuric acid etching recorded a mean retention value of $(87.45 \mathrm{~N})$. However, the results were statistically non-significant.

These obtained data support the previously postulated hypothesis for this study, where different surface treatment protocols showed comparable results.

The results of our study were comparable with the results of Uhrenbacher et al. ${ }^{(18)}$ Who concluded that retention of PEEK crowns after acid etching with sulphuric acid or sandblasting showed a highly satisfactory results as the surface energy of the PEEK was increased after acid etching and sandblasting. In addition acid etching increases the surface polarity when the oxygen in sulfuric acid reacts with and breaks the PEEK benzene ring so that more functional groups show bonding potential.

Also the results were in accordance with Stawarczyk et al. ${ }^{(15)}$ who concluded that cementation of PEEK crowns by a methacrylate containing adhesive after surface treatment with airborn abraision by alumina 110 micron or acid etching with sulphuric acid showed a high bond strength due to increase in actual surface area in a roughened surface compared to a smooth surface, allowing a solid to attract more medium, and a higher inter- facial force can be produced.

\section{REFERENCES}

1. Ausiello, P., Rengo, S., Davidson, C. L., \& Watts, D. C.: (2004): Stress distributions in adhesively cemented ceramic and resin-composite class II inlay restorations: A 3D-FEA study: Dent. Mater., 20(9), 862-872. https://doi. org/10.1016/j.dental.2004.05.001.

2. Bosch, G., Ender, A., \& Mehl, A.: (2014): A 3-dimensional accuracy analysis of chairside CAD/CAM milling processes: J. Prosthet. Dent., 112(6), 1425-1431. https:// doi.org/10.1016/j.prosdent.2014.05.012.

3. Goracci, C., Cury, A. H., Cantoro, A., Papacchini, F., Tay, F. R., \& Ferrari, M.: (2006): Microtensile bond strength and interfacial properties of self-etching and self-adhesive resin cements used to lute composite onlays under different seating forces.: J. Adhes. Dent., 8(5), 327-335. http:// www.ncbi.nlm.nih.gov/pubmed/17080881.
4. Hallmann, L., Mehl, A., Sereno, N., \& Hämmerle, C. H. F. (2012): The improvement of adhesive properties of PEEK through different pre-treatments: Appl. Surf. Sci., 258(18), 7213-7218. https://doi.org/10.1016/j.apsusc.2012.04.040.

5. Heintze, S. D.: (2010): Crown pull-off test (crown retention test) to evaluate the bonding effectiveness of luting agents: Dent. Mater., 26(3), 193-206. https://doi.org/10.1016/j. dental.2009.10.004

6. Johnson, G. H., Lepe, X., Patterson, A., \& Schäfer, O.: (2018): Simplified cementation of lithium disilicate crowns: Retention with various adhesive resin cement combinations: J. Prosthet. Dent., 119(5), 826-832. https:// doi.org/10.1016/j.prosdent.2017.07.012.

7. Karimipour-Saryazdi, M., Sadid-Zadeh, R., Givan, D., Burgess, J. O., Ramp, L. C., \& Liu, P. R.: (2014): Influence of surface treatment of yttrium-stabilized tetragonal zirconium oxides and cement type on crown retention after artificial aging: J. Prosthet. Dent., 111(5), 395-403. https:// doi.org/10.1016/j.prosdent.2013.09.034

8. Keul, C., Liebermann, A., Schmidlin, P. R., Roos, M., Sener, B., \& Stawarczyk, B.: (2014): Influence of PEEK surface modification on surface properties and bond strength to veneering resin composites: J. Adhes. Dent., 16(4), 383-392. https://doi.org/10.3290/j.jad.a32570

9. Kirsch, C., Ender, A., Attin, T., \& Mehl, A.: (2017): Trueness of four different milling procedures used in dental CAD/CAM systems: Clin. Oral Investig., 21(2), 551-558. https://doi.org/10.1007/s00784-016-1916-y

10. Kurtz, S. M., \& Devine, J. N.: (2007): PEEK biomaterials in trauma, orthopedic, and spinal implants: Biomaterials, 28(32), 4845-4869. https://doi.org/10.1016/j.biomaterials.2007.07.013.

11. Najeeb, S., Zafar, M. S., Khurshid, Z., \& Siddiqui, F.: (2016a): Applications of polyetheretherketone (PEEK) in oral implantology and prosthodontics: J. Prosthodont. Res., 60(1), 12-19. https://doi.org/10.1016/j.jpor.2015.10.001.

12. Sadighpour, L., Geramipanah, F., Fazel, A., Allahdadi, M., \& Kharazifard, M.J.: (2018): Effect of Selected Luting Agents on the Retention of CAD/CAM Zirconia Crowns Under Cyclic Environmental Pressure.: J. Dent. (Tehran)., 15(2), 97-105. http://www.ncbi.nlm.nih.gov/ pubmed/29971127\%0Ahttp://www.pubmedcentral.nih. gov/articlerender.fcgi?artid=PMC6026310.

13. Silva Casado, B.G. da: (2017): Bond Strength between Zirconia Y-TZP and Resin Cements: Evaluation of Sur- 
face Treatment, Cure Mode, and Failure Types: J. Dent. Heal. Oral Disord. Ther., 8(5), 582-586. https://doi. org/10.15406/jdhodt.2017.08.00296.

14. Son, Y. H., Han, C. H., \& Kim, S.: (2012): Influence of internal-gap width and cement type on the retentive force of zirconia copings in pullout testing: J. Dent., 40(10), 866-872. https://doi.org/10.1016/j.jdent.2012.07.007.

15. Stawarczyk, B., Beuer, F., Wimmer, T., Jahn, D., Sener, B., Roos, M., \& Schmidlin, P. R.: (2013): Polyether ether ketone - A suitable material for fixed dental prostheses? J. Biomed. Mater. Res. - Part B Appl. Biomater., 101(7), 1209-1216. https://doi.org/10.1002/jbm.b.32932

16. Stawarczyk, B., Eichberger, M., Uhrenbacher, J., Wimmer, T., Edelhoff, D., \& Schmidlin, P. R.: (2015): Threeunit reinforced polyetheretherketone composite FDPs: Influence of fabrication method on load-bearing capacity and failure types: Dent. Mater. J., 34(1), 7-12.

17. Tekin, S., Cangül, S., Adıgüzel, Ö., \& Değer, Y.: (2018): Areas for use of PEEK material in dentistry: Int. Dent. Res., 8(2), 84-92. https://doi.org/10.5577/intdentres.2018. vol8.no2.6
18. Uhrenbacher, J., Schmidlin, P. R., Keul, C., Eichberger, M., Roos, M., Gernet, W., \& Stawarczyk, B.: (2014): The effect of surface modification on the retention strength of polyether ether ketone crowns adhesively bonded to dentin abutments: J. Prosthet. Dent., 112(6), 1489-1497. https:// doi.org/10.1016/j.prosdent.2014.05.010

19. Vinaya, K., Rakshith, H., Krishna, P. D., Manoj, S., Mankar, S., \& Shetty, N.: (2015): To evaluate \& compare retention of complete cast crown in natural teeth using different auxiliary retentive features with two different crown heights - An in vitro study: Int. J. Biomed. Sci., 11(2), 99-106.

20. Xie, H., Tay, F. R., Zhang, F., Lu, Y., Shen, S., \& Chen, C.: (2015): Coupling of 10-methacryloyloxydecyl dihydrogen phosphate to tetragonal zirconia: Effect of $\mathrm{pH}$ reaction conditions on coordinate bonding: Dent. Mater., 31(10), e218-e225. https://doi.org/10.1016/j.dental.2015.06.014

21. Yousry, M. A., Hussein, S. A., \& Al Abbassy, F. H.: (2018): Evaluation of Shear Bond Strength of High-Performance Polymers To Its Resin Veneering and To Dentin (in Vitro Study): Alexandria Dent. J., 43(2), 62-68. https://doi. org/10.21608/adjalexu.2018.57626. 